\title{
THE DEVELOPMENT OF A DIRECTED POPULATION APPROACH TO TACKLE INEQUALITIES IN DENTAL CARIES PREVALENCE AMONG SECONDARY SCHOOL CHILDREN BASED ON A SMALL AREA PROFILE
}

\author{
Darius Sagheri ${ }^{1}$, Petra Hahn ${ }^{2}$, Elmar Hellwig ${ }^{2}$ \\ ${ }^{1}$ Department of Orthodontics, School of Dental Science, University of Cologne, Germany \\ 2Department of Restorative Dentistry and Periodontology, Albert Ludwigs University of Freiburg, Germany
}

\section{SUMMARY}

Background: It has been observed that the prevalence of dental caries among children has declined in the last decade in Germany. However, despite of these improvements there is still a proportion of children suffering from dental decay.

Aims: The aims of this study were to evaluate if a social gradient in the prevalence of dental caries exists and, based on those findings, to develop a strategy to target those children with heightened risk to develop dental caries in order to assist oral health care professionals to refocus the current uniform school-based dental health programme to a caries preventive strategy based on a directed population approach.

Design: A representative, random sample of 12-year olds in Freiburg (Germany) was examined and dental caries was recorded using WHO criteria. Educational attainment of the child's parents was used as an indicator of socio-economic status and classified by use of the CASMIN Educational Classification.

Results: A total of 322 children participated. An examination of dental caries score revealed that its distribution was positively skewed. For this reason this study provides summary analyses based on medians and a non-parametric rank-sum test. The Kruskal-Wallis H-test showed a significant difference between median scores across the different educational levels ( $p$-value $=0.015)$ which was due to lower dental caries levels in children with non-deprived social background.

Conclusions: In order to reduce current social inequalities in child oral health the current uniform school-based dental health programme at secondary school level should be developed to a targeted school-based screening and prevention programme.

Key words: children, dental caries, educational level, Germany, inequalities, oral health

Address for correspondence: D. Sagheri, Department of Orthodontics, School of Dental Science, University of Cologne, Kerpener Strasse 32, 50931 Cologne, Germany. E-mail: darius.sagheri@uk-koeln.de

\section{INTRODUCTION}

The prevalence of dental caries among children and adolescents in industrialized countries has declined in the last two decades (1). Survey data on children's oral health collected in Germany demonstrated that dental caries levels in Germany followed this trend $(2,3)$.

In Germany responsibilities for health and education are held by the 16 states and therefore may vary from state to state. However, in general, state salaried dentists, who are often part-time employees with a background in family or paediatric dentistry, perform uniform annual dental examination and may provide on a second visit oral health education and/or the application of fluoride varnish for schoolchildren. These school-based oral health programmes are provided by the dental section of the public health service at city or county level in all state maintained pri- mary and secondary schools. If a child is found to require dental treatment the examining dentist will issue an information note for the legal guardian. It is the legal guardian's responsibility to make all necessary arrangements with a dentist in private practice to provide dental treatments which are in most cases paid for by a statutory health insurance and are free for the recipient at the point of delivery.

The German education system provides different paths for students based on individual ability. All children attend primary school until fourth standard (i.e. up to 10 years of age). After that they can take one of three school paths ('Hauptschule', 'Realschule', 'Gymnasium') based on their school performance. 'Hauptschule' is supposed to prepare mainly practically oriented students for craft professions. 'Realschule' is supposed to prepare mainly practically and theoretically oriented students for trade, technical and administrative professions. 'Gymnasium' caters 
mainly for theoretically oriented students, who are to be prepared for various studies at universities.

Despite of the improvements in oral health at population level in the last two decades there is still a proportion of the child population suffering from dental caries and child oral health surveys demonstrated repeatedly a social gradient in the prevalence of dental caries in Germany (2, 3) and elsewhere (4). Mielck and Bloomfield (5) demonstrated that it makes little difference whether socio-economic position is defined by parental educational level, income or profession, but when one of these socio-economic parameter is linked together with oral health data a social gradient becomes apparent (4). However, difficulties arise in translating this knowledge on unequal distribution of oral health to a workable strategy for oral health professionals in order to target children with heightened need for dental treatment and increased risk of developing dental decay.

The aims of this study were to evaluate at community level if a social gradient in the prevalence of dental caries in representative sample of school-age children in Freiburg (Germany) exists and, based on those findings, to develop a strategy to target those children with heightened risk to develop dental caries in order to assist oral health care professionals in the public health service to refocus the current uniform school-based dental health programme to a caries preventive strategy based on a directed population approach.

\section{MATERIAL AND METHODS}

\section{Sample}

Selection for age in the sample was carried out on the basis of the children's standard (class) in school. Second standard secondary school was selected to represent 12-year olds. Children aged 12 years were selected as the study population because children in this age group have recently been graded into one of the three school strata ('Hauptschule', 'Realschule', 'Gymnasium'). Furthermore, survey data collected on this age-group is comparable with earlier German studies and with studies conducted internationally as this age group was recommended for study by the World Health Organisation (WHO).

The District Education Department in Freiburg (total population of approximately 205,000, had a population of 12-year olds of approximately 1,600 (6)) provided information on numbers of children in the classes and schools. This permitted determination of the sample size and the random selection of schools for inclusion in the study. The total target sample size required, based on a confidence level of $95 \%$, margin of error of $5 \%$, was 310 children. The study included all state-maintained schools with 12-year olds on the school roll. The primary sampling unit was the school. The second stage of the sampling process was then the selection of one class. If a school had more than one class available each class has an equal probability of being randomly selected. All children within a class were included in the sample. A list of children in each class was obtained from the selected schools. This selection process continued until sufficient numbers of children were obtained.

\section{Ethical Approval and Consent}

The department of education at the regional state government administration reviewed the ethical and legal aspects of the research protocol and approved the study. All parents of participating children were fully informed regarding the nature of the study and the benefits of participating. Clinical examinations were only carried out in the children with completed consent forms ('Positive Consent').

\section{Social Stratification}

Educational attainment is a widely used indicator of socioeconomic status in health studies $(7,8)$. The use of education based cut-offs represents a simple, clinically applicable decision rule. Questions about education are less sensitive than questions about family income and are more reliably reported (9). The CASMIN Educational Classification $(10,11)$, which is one of the standard approaches to record educational level in social science, was selected as a classification because it is applicable in various countries and may allow researchers to compare findings of this study with a wider range of studies. The CASMIN Educational Classification is available for France, Germany, Hungary, Ireland, Italy, Netherlands, Spain, Sweden and United Kingdom. The CASMIN Educational Classification consists of a coding schema that differentiated between nine levels of educational qualifications. These nine levels can be collapsed to fit researchers' needs into a threefold hierarchical division which could be equally well taken as ordering positions in terms of their socioeconomic status. Parental educational attainment was recorded by use of a questionnaire.

\section{Training and Calibration of the Examiner}

The examiner (DS) and recorder (dental surgery assistant) were trained and calibrated prior to the commencement of the fieldwork to ensure reliability on the measurement index following WHO guidelines $(12,13)$. The trainer, a senior lecturer in public dental health at the WHO Collaborating Centre for International Collaboration in Dental Education with extensive experience in oral health surveys, provided the standard against which the examiner (DS) was calibrated. The level of agreement between the examiner and the gold standard was assessed by use of the Kappa statistic, as described by Fleiss and Chilton (14). A validation exercise was conducted during the fieldwork (re-examination of $10 \%$ of the subjects) to monitor examiner agreement and consistency during the course of the survey.

\section{Fieldwork and Data Processing}

Dental caries was recorded at DMFT level i.e. when clinically detectable lesions in dentine and lesions into the pulp were evident using WHO criteria (12). All children were examined in their schools under the same standardised conditions. A transportable halogen lamp [Daray Versatile Medical Light with halogen bulb, 12 volts, 20 watts (Daray Ltd., Swadlincote, UK)] was used to illuminate the mouth and clamped to the table in a position that enabled it to be angled towards the mouth. The teeth were examined visually for dental caries. The teeth were examined wet and a CPITN probe was only used to confirm a diagnosis of cavitation or to remove food debris. Data were collected on schematised charts and processed using the Statistical Package for the Social Sciences, SPSS 12 (SPSS, Inc., Chicago, USA). 


\section{RESULTS}

Examiner and recorder were calibrated to ensure reliability of the measurement index used prior to the commencement of the fieldwork. The level of agreement between the examiner and the gold standard was calculated and showed an agreement of Kappa 0.87 . A validation exercise was conducted during the fieldwork (re-examination of $10 \%$ of the subjects) to monitor examiner agreement and consistency during the course of the survey (intraexaminer Kappa 0.89).

In all, 13 secondary schools in Freiburg were included in the sample. Three hundred and seventy-eight (378) consent forms and questionnaires were issued. A total of 322 (85\% response) completed consent forms were returned and subsequently the same number of children was examined. The difference between the numbers of issued consent forms and questionnaires and the examined children is mostly made up of children who were not present on the day of the examination or who forgot to return the consent forms. Out of these 164 females (50.9\%) and 158 males (49.1\%) were examined. The mean age of the children on the day of examination was 11.66 .

The data (Table 1) are presented as the mean values of decayed, missing and filled permanent teeth (DMFT). The mean DMFT for the sample was 0.69 (1.19 standard deviation). The proportion of children with caries-free dentition (DMFT $=0$ ) was $66.1 \%$.

In order to get a further insight of the distribution of dental caries in the child population in Freiburg the DMFT scores were stratified into three levels of CASMIN educational qualifications as a proxy for social stratification. Two hundred and ninety-four (294) parents of the 322 children (91.3\%) answered questions concerning their educational qualification. Table 1 shows the DMFT mean values for each CASMIN level. The mean DMFT

Table 1. Mean DMFT (SD), proportion of caries free children and SiC Index score for the total sample and Kruskal-Wallis mean rank; mean DMFT (SD), number of children contributing to SiC Index and their proportion and the proportion of caries free children for each CASMIN level

\begin{tabular}{|l|c|c|c|}
\hline Variable & \multicolumn{3}{|c|}{ Dental Caries Level } \\
\hline $\mathrm{n}$ & \multicolumn{3}{|c|}{0.69} \\
\hline Mean DMFT & \multicolumn{3}{|c|}{1.19} \\
\hline Std. Deviation & \multicolumn{3}{|c|}{$66.1 \%$} \\
\hline Caries free (\%) & \multicolumn{3}{|c|}{} \\
\hline SiC Index score & \multicolumn{3}{|c|}{} \\
\hline $\begin{array}{l}\text { CASMIN } \\
\text { (parents educational } \\
\text { level) }\end{array}$ & $\begin{array}{c}\text { CASMIN 1 } \\
\text { (lowest level) }\end{array}$ & $\begin{array}{c}\text { CASMIN 2 } \\
\text { (medium level) }\end{array}$ & $\begin{array}{c}\text { CASMIN 3 } \\
\text { (highest } \\
\text { level) }\end{array}$ \\
\hline $\mathrm{n}$ & 88 & 107 & 99 \\
\hline Kruskal-Wallis Rank & 165.18 & 143.36 & 136.27 \\
\hline Mean DMFT & 1.0 & 0.58 & 0.45 \\
\hline Std. Deviation & 1.46 & 1.06 & 0.96 \\
\hline $\mathrm{n}$ (SiC Index) & 38 & 32 & 26 \\
\hline $\begin{array}{l}\text { Proportion of SiC } \\
\text { group (\%) }\end{array}$ & 43.7 & 30.0 & 26.3 \\
\hline Caries free (\%) & 56.3 & 70.1 & 73.7 \\
\hline
\end{tabular}

for CASMIN 1 (lowest educational level) was 1.0, for CASMIN 2 (medium educational level) it was 0.58 and for CASMIN 3 (highest educational level) it was 0.45 . As it is typical in low caries populations the DMFT values are not normally distributed but are positively skewed with the result that reporting the mean DMFT of the sample population gives an incomplete picture of the distribution of dental caries in that population. For this reason the present study provides summary analyses based on medians, inter-quartile range and a non-parametric rank sum test and on the 'Significant Caries Index, SiC Index' (15).

Analysis based on medians and inter-quartile range and the non-parametric Kruskal-Wallis H-test showed a significant difference between median scores across the different CASMIN levels. The association between the DMFT scores and the different CASMIN levels is considered to be statistically highly significant (the 1 -way $\chi^{2}$ equals 8.381 ; the p-value equals 0.015 ). The significance is due to CASMIN 1 distribution differing markedly from CASMIN 2 and CASMIN 3 (Fig. 1).

The SiC Index is the mean DMFT of the one third of the study group with the highest caries scores. In Freiburg the SiC Index score was 2.01 (Table 1). When the group of children who contribute to the $\mathrm{SiC}$ Index was further analysed and the proportion of children from each CASMIN level determined it can be seen that a social gradient becomes apparent. Children with CASMIN 1 background represent the largest group of children (43.7\%) contributing to the $\mathrm{SiC}$ Index when compared with their peers with CASMIN 2 (30.0\%) and CASMIN 3 (26.3\%) backgrounds. Examination of the numbers of caries free children for each

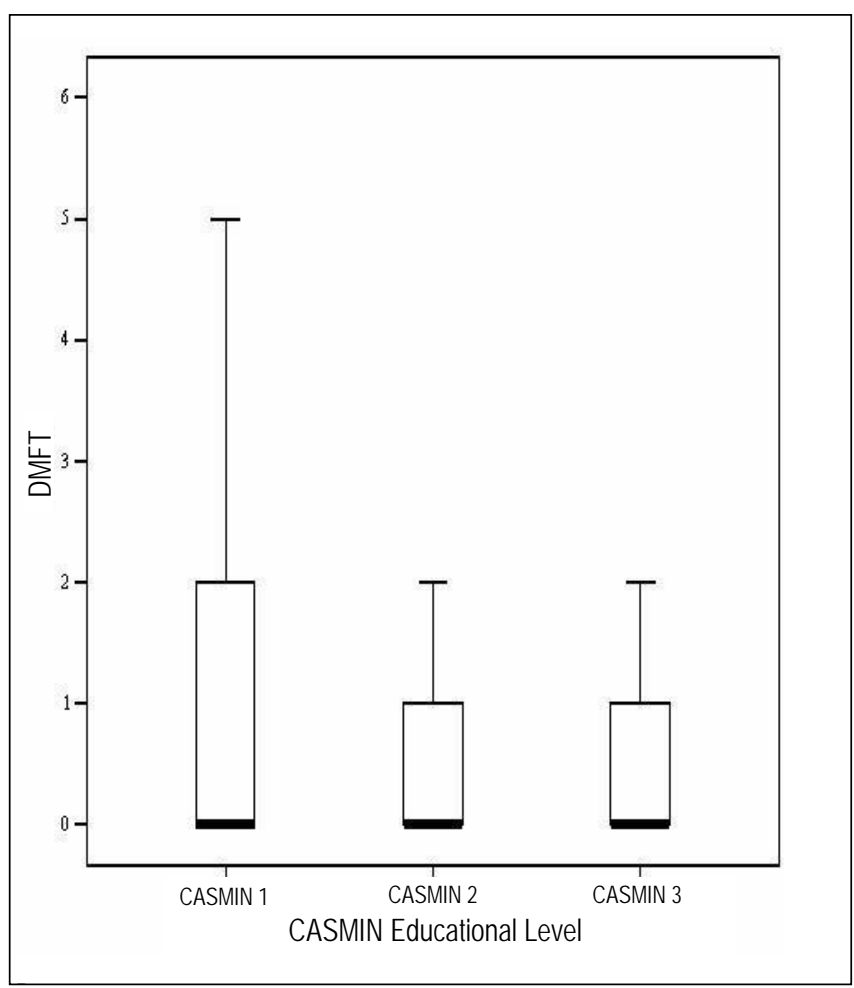

Fig. 1. Boxplot of the DMFT distribution for each CASMIN level. The boxplot allows comparing the different median scores for the three educational levels and their variation (length of the box, which represents the middle $50 \%$ of the DMFT scores, and whiskers, which represents the range of the DMFT scores). 
Table 2. Frequency distribution of children in each school strata (Hauptschule, Realschule, Gymnasium) arranged by their parent's educational level (CASMIN) and the proportion of children within each school strata who contributed to the SiC Index

\begin{tabular}{|c|c|c|c|c|}
\hline & & \multicolumn{3}{|c|}{ Children's School Strata } \\
\hline & & $\begin{array}{c}\text { Hauptschule } \\
\text { (Basic academic level) }\end{array}$ & $\begin{array}{c}\text { Realschule } \\
\text { (Medium academic level) }\end{array}$ & $\begin{array}{c}\text { Gymnasium } \\
\text { (Medium academic level) }\end{array}$ \\
\hline & $n$ & 65 & 92 & 137 \\
\hline \multirow{3}{*}{ 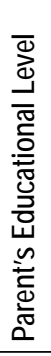 } & $\begin{array}{l}\text { CASMIN } 1 \\
\text { (Lowest level) }\end{array}$ & $\begin{array}{l}64.6 \% \\
(n=42)\end{array}$ & $\begin{array}{l}35.9 \% \\
(n=33)\end{array}$ & $\begin{array}{c}9.5 \% \\
(n=13)\end{array}$ \\
\hline & $\begin{array}{l}\text { CASMIN } 2 \\
\text { (Medium level) }\end{array}$ & $\begin{array}{l}24.6 \% \\
(n=16)\end{array}$ & $\begin{array}{l}51.1 \% \\
(n=47)\end{array}$ & $\begin{array}{l}32.1 \% \\
(n=44)\end{array}$ \\
\hline & $\begin{array}{l}\text { CASMIN } 3 \\
\text { (Highest level) }\end{array}$ & $\begin{array}{l}10.8 \% \\
(n=7)\end{array}$ & $\begin{array}{l}13.0 \% \\
(n=12)\end{array}$ & $\begin{array}{l}58.4 \% \\
(n=80)\end{array}$ \\
\hline \multirow{2}{*}{$\begin{array}{l}\frac{x}{0} \\
\frac{0}{\leq} \\
\text { 心 }\end{array}$} & $\mathrm{n}$ & 33 & 41 & 35 \\
\hline & $\begin{array}{l}\text { Proportion of children } \\
\text { within each school strata }\end{array}$ & $40.2 \%$ & 40.6 & 25.1 \\
\hline
\end{tabular}

CASMIN level revealed an opposite trend, which reconfirmed previous findings. Children with CASMIN 3 background represent the largest proportion of children with caries free dentition (73.7\%) when compared with children with CASMIN 2 (70.1\%) and CASMIN 3 (56.3\%) backgrounds.

In order to enable public health administrators and professionals to apply these findings and to develop a targeted school-based oral health prevention strategy it was decided to investigate if a correlation between socio-economic status (i.e. expressed by parental educational level) and the enrolment patterns of their children in the three different school strata (Hauptschule, Realschule, Gymnasium) at secondary school level exists. Table 2 demonstrates that an abundant proportion of children enrolled in Hauptschule have parents with a CASMIN 1 background (64.1\%), children enrolled in Realschule have a considerable proportion of parents with a CASMIN 2 background (51.1\%) and children enrolled in Gymnasium have an ample proportion of parents with a CASMIN 3 background (58.1\%). In order to investigate this distribution pattern further the Spearman correlation coefficient was calculated. The correlation coefficient equals 0.54 and is considered to be statistically highly significant (the $\mathrm{p}$-value $<0.001$ ). Further analysis based on the group of children who contribute to the SiC Index and the school strata they attend (Table 2) reconfirmed the existence of a social gradient: from all children who attend Hauptschule and Realschule an almost equally large group contributed to the SiC Index (40.2\% and $40.6 \%$ respectively) whereas only $25.1 \%$ of all children who attend Gymnasium contributed to the $\mathrm{SiC}$ Index.

\section{DISCUSSION}

The present study demonstrates the existence of social gradient in child oral health in a population with overall low dental caries levels. It was decided to use parents' educational level as a proxy for social stratification because it has been demonstrated that it makes little difference whether socio-economic position is defined by parental educational level, income or profession (5), but educational level seems to yield a better response than other socio-demographic parameter (9). The present study demonstrated that children from lower socio-economic groups have considerably higher dental caries levels than their peers from higher socio-economic groups. However, at present no comprehensive, all accepted single explanation for social inequalities in health and oral health exists (16). Various explanations for inequalities in oral health have been put forward which vary in their focus from the effects of material deprivation, to individual lifestyle decisions (17). Health inequalities may well result from the interaction of material, behavioural and psychosocial factors over time (18). For example, a materialist explanation may demonstrate a rationale for some of the existing inequalities. Evidence shows that within industrialized societies lower socio-economic groups purchase higher amounts of sugars, preserves and refined carbohydrates (19), which are risk factors for the development of dental caries (20), than higher socio-economic groups, because these foods are cheaper and more filling and therefore representing good value for money for low income families. Healthier diets, containing higher amounts of fruit and vegetables, are more pricy and may therefore be out of reach for low-income families (21).

Thompson and co-workers (22) found strong evidence that adult oral health can be predicted by childhood socio-economic status and oral health. Therefore, tackling inequalities in dental caries prevalence in children and adolescents, even in a low dental caries population, may result in long lasting benefits which reach into adulthood. Because resources for school-based oral health programmes are limited and have been cut in recent times, it has been recommended that they should target those sections of the population who would most benefit from an oral health prevention programme. In the Freiburg region, school children are provided with uniform annual dental examination and on a second visit with oral health education which may be combined with the application of fluoride varnish. However, so far no strategy has been 
put forward to develop the current uniform German school-based dental screening and education programme further to a targeted programme which is linked to a socio-economic indicator.

The present study demonstrated a robust link between socioeconomic position, expressed in educational attainment of parents, and children's enrolment patterns at secondary school level. Children with a low socio-economic background tend to populate Hauptschule whereas children with a medium socio-economic background predominantly attend Realschule and children with a high socio-economic background mainly attend Gymnasium. This finding demonstrates that the three school strata at secondary school level can be used as a proxy for social stratification and can serve as a useful marker to classify schools into risk strata and therefore can form the basis for a targeted school-based oral health prevention strategy at secondary school level. However, Batchelor and Sheiham (23) pointed out that a preventive programme has still to be aimed at the whole population because a considerable proportion of new caries lesions occur in individuals who would not be reached by programmes that were confined to high-risk children only. Furthermore, research from Finland showed that application of a high-risk strategy did not improve the dental health among high caries risk children substantially when compared to a similar group who received basic prevention only (24). Therefore, in order to avoid that high-risk children who attend low-risk schools are missed because of the targeted approach, a screening circle should still be employed in low-risk school rather than ceasing all screenings. In Freiburg, all children should still be provided with annual school-based screenings but only children who attend Hauptschule and Realschule should be provided with additional school-based preventive measures. Most children with dental needs who attend schools classified as low-risk (i.e. Gymnasium) will still receive dental care, since they tend to have a higher socio-economic background and therefore are most likely to avail or already have a history of using dental services (25).

Sköld and co-workers (26) showed in their study on the effect of school-based fluoride varnish programmes that those programmes had a great impact on dental caries levels in children with medium and high-risk of development of dental caries. They demonstrated that bi-annual treatments at 6-month intervals were most cost-effective. Seppä and Tolonen (27) showed that fluoride varnish application performed more frequently than twice a year may not provide additional caries protection in a population with a relatively low caries incidence rate. The provision of fluoride varnish at school level is effective particularly in socially deprived areas, because no arrangements, such as appointments for prophylactic dental treatment, need to be made and therefore can not be missed (28). Therefore, schools identified as to benefit from additional school-based preventive measures (i.e. Realschule and Hauptschule) should be provided in addition to school screenings with bi-annual fluoride varnish treatments at 6-month intervals. The concentration of public dental health efforts for low-risk schools (i.e. Gymnasium) to annual school-based dental screenings will free up needed resources to provide these additional preventive measure.

Locker and co-workers (25) found in their evaluation of a targeted school-based dental screening programme that the majority of children from lower socio-economic background with dental care needs were identified by a targeted screening programme and were the main beneficiaries of such a targeted programme. Furthermore, evaluations of such programmes demonstrated that they are effective in increasing dental attendance rates among children from lower socio-economic groups and areas (24). Sköld et al. (26) showed that a targeted schoolbased screening and prevention programme is of importance at secondary school level because children in this age-group have the highest number of caries free permanent approximal tooth surfaces but these surfaces will become the predominant surface for dental treatment when adolescents go through various life course transitions. Furthermore, they demonstrated in their study that the application of fluoride varnish is an excellent tool to prevent approximal caries in 13- to 16-year olds with medium and high caries risk. Other recent Swedish research showed that fewer new approximal enamel lesions develop during young adulthood than during adolescence (29), which underpins the importance of a targeted school-based screening and prevention programme at secondary school level.

To conclude, in order to reduce current social inequalities in child and adolescence oral health in Freiburg the current uniform school-based screening and education programme at secondary school level should be developed to a targeted school-based screening and prevention programme with annual screenings for all school strata and additional bi-annual fluoride varnish applications at 6-month intervals at Realschule and Hauptschule level.

\section{REFERENCES}

1. Marthaler TM, O’Mullane DM, Vrbic V. The prevalence of dental caries in Europe 1990-1995. ORCA Saturday afternoon symposium 1995. Caries Res. 1996;30(4):237-55.

2. Schulte AG, Momeni A, Pieper K. Caries prevalence in 12-year-old children from Germany. Results of the 2004 national survey. Community Dent Health. 2006 Dec;23(4):197-202.

3. Pieper K, Schulte AG. The decline in dental caries among 12-year-old children in Germany between 1994 and 2000. Community Dent Health. 2004 Sep;21(3):199-206.

4. Locker D. Deprivation and oral health: a review. Community Dent Oral Epidemiol. 2000 Jun;28(3):161-9.

5. Mielck A, Bloomfield K. Reducing income inequity and boosting social capital: new responsibilities in social epidemiological research. Gesundheitswesen. 2001 Mar;63 Suppl 1:S18-23. (In German.)

6. City of Freiburg. Official Census Data since 1950. Freiburg: Amt für Bürgerservice und Informationsverarbeitung; 2006. (In German.)

7. Osler M, Prescott E. Educational level as a contextual and proximate determinant of all cause mortality in Danish adults. J Epidemiol Community Health. 2003 Apr;57(4):266-9.

8. Hardarson T, Gardarsdóttir M, Gudmundsson KT, Thorgeirsson G, Sigvaldason H, Sigfússon N. The relationship between educational level and mortality. The Reykjavík Study. J Intern Med. 2001 Jun;249(6):495502.

9. Fiscella K, Franks P. Should years of schooling be used to guide treatment of coronary risk factors? Ann Fam Med. 2004 Sep-Oct;2(5):469-73.

10. Braun M, Müller W. Measurement of education in comparative research. Comp Soc Res. 1997;16:163-201.

11. König W, Lüttinger P, Müller W. A comparative analysis of the development and structure of educational systems. Methodological foundations and the construction of a comparative educational scale. CASMIN working paper no. 12. Mannheim: University of Mannheim; 1988.

12. World Health Organization. Oral health surveys: basic methods. 4th ed. Geneva: WHO; 1997.

13. Eklund SA, Moller IJ, Leclercq MH. Calibration of examiners for oral health epidemiological surveys. Geneva: WHO; 1993.

14. Fleiss JL, Chilton NW. The measurement of interexaminer agreement on periodontal disease. J Periodontal Res. 1983 Nov;18(6):601-6. 
15. Bratthall D. Introducing the Significant Caries Index together with a proposal for a new global oral health goal for 12-year-olds. Int Dent J. 2000 Dec;50(6):378-84.

16. Marmot M. Social determinants of health inequalities. Lancet. $2005 \mathrm{Mar}$ 19-25;365(9464):1099-104.

17. Sisson KL. Theoretical explanations for social inequalities in oral health. Community Dent Oral Epidemiol. 2007 Apr;35(2):81-8.

18. Blane D. The life course, the social gradient, and health. In: Marmot M, Wilkinson R, editors. Social determinants of health. Oxford: Oxford University Press; 1999. p. 64-80.

19. Turrell G, Kavanagh AM. Socio-economic pathways to diet: modelling the association between socio-economic position and food purchasing behaviour. Public Health Nutr. 2006 May;9(3):375-83.

20. Rugg-Gunn AJ, Edgar WM. Sugar and dental caries: a review of the evidence. Community Dent Health. 1984 Jul;1(2):85-92.

21. Drewnowski A, Specter SE. Poverty and obesity: the role of energy density and energy costs. Am J Clin Nutr. 2004 Jan;79(1):6-16.

22. Thomson WM, Poulton R, Milne BJ, Caspi A, Broughton JR, Ayers KM. Socioeconomic inequalities in oral health in childhood and adulthood in a birth cohort. Community Dent Oral Epidemiol. 2004 Oct;32(5):34553.

23. Batchelor PA, Sheiham A. The distribution of burden of dental caries in schoolchildren: a critique of the high risk caries prevention strategy for populations. BMC Oral Health. 2006 Jan 31;6(1):3.
24. Hausen H, Kärkkäinen S, Seppä L. Application of the high-risk strategy to control dental caries. Community Dent Oral Epidemiol. 2000 Feb;28(1):26-34.

25. Locker D, Frosina C, Murray H, Wiebe D, Wiebe P. Identifying children with dental care needs: evaluation of a targeted school-based dental screening program. J Public Health Dent. 2004;64(2):63-70.

26. Sköld UM, Petersson LG, Lith A, Birkhed D. Effect of school-based fluoride varnish programmes on approximal caries in adolescents from different caries risk areas. Caries Res. 2005;39(4):273-9.

27. Seppä L, Tolonen T. Caries preventive effect of fluoride varnish applications performed two or four times a year. Scand J Dent Res. 1990 Apr;98(2):102-5.

28. Zimmer S, Robke FJ, Roulet JF. Caries prevention with fluoride varnish in a socially deprived community. Community Dent Oral Epidemiol. 1999 Apr;27(2):103-8.

29. Mejàre I, Stenlund H, Zelezny-Holmlund C. Caries incidence and lesion progression from adolescence to young adulthood: a prospective 15-year cohort study in Sweden. Caries Res. 2004 Mar-Apr;38(2):130-41.

Received December 18, 2007 Accepted February 12, 2008 